\title{
Osteítis por radiación y fracturas por insuficiencia pélvica en paciente con adenocarcinoma de próstata tratado con radioterapia. Aspectos clínicos y radiográficos de la enfermedad.
}

\author{
DOI: http//dx.doi.org/10.37315/SOTOCAV20202835591
}

FERNÁNDEZ GABARDA $R^{1}$, BALLESTA MORATALLA $M^{2}{ }^{2}$, SANGÜESA NEBOT MJ ${ }^{1}$.

(1) SERVICIO DE TRAUMATOLOGÍA Y CIRUGÍA ORTOPÉDICA. HOSPITAL ARNAU DE VILANOVA DE VALENCIA

(2) SERVICIO DE RADIODIAGNÓSTICO. HOSPITAL UNIVERSITARIO Y POLITÉCNICO LA FE. VALENCIA

\section{Resumen.}

Osteítis por radiación es un término anatomopatológico acuñado por primera vez por Ewing en 1926 y hace referencia a una respuesta inflamatoria inducida por la radiación en los vasos sanguíneos, el tejido nervioso y las células óseas que pueden conducir a fracturas del hueso trabecular y cortical. La fractura por insuficiencia pélvica es una fractura de stress sobre un hueso debilitado, visto en pacientes que tienen ciertos factores de riesgo como osteoporosis, enfermedades del tejido conectivo, uso de corticoides y sobre todo en pacientes que han sido tratados con radioterapia pélvica por tumores rectales, anales, urogenitales y de próstata. Presentamos el caso clínico de un paciente de 73 años, con fracturas por insuficiencia pélvica tratado cuatro años antes de adenocarcinoma de próstata mediante radioterapia y hormonoterapia. Se discuten aspectos clínicos y radiográficos diagnósticos.

Palabras clave: osteítis por radiación, fractura por insuficiencia, radioterapia, adenocarcinoma próstata.

\section{Summary.}

Radiation osteitis is an anatomopathological term first coined by Ewing in 1926, refers to a radiation-induced inflammatory response in blood vessels, nerve tissue, and bone cells that can lead to fractures of the trabecular and cortical bone. Pelvic insufficiency fracture is a stress fracture over a weakened bone, seen in patients who have certain risk factors such as osteoporosis, connective tissue disease, use of corticosteroids, and especially in patients who have been treated with pelvic radiation therapy for rectal, anal, urogenital, and prostate tumors. We present the case of a 73-year-old patient with fractures due to pelvic failure treated four years before prostate adenocarcinoma with radiotherapy and hormone therapy. Clinical and radiographic diagnostic aspects are discussed.

\author{
Correspondencia: \\ Rafael Fernández Gabarda. \\ e-mail: raferga@hotmail.com
}




\section{Introducción}

Los cambios histológicos que la radiación provoca en el hueso se conocen como osteítis por radiación. Fueron inicialmente descritos por Ewing en $1926^{1}$, y son el sustrato anatomopatológico de la fractura por insuficiencia.

La fractura por insuficiencia es un tipo de fractura de stress que se produce cuando se aplican fuerzas fisiológicas sobre un hueso debilitado y con una resistencia elástica disminuida. Es diferente de la fractura por fatiga, que se produce en un hueso normal ante microtraumatismos repetitivos $^{2}$

El sacro es el hueso más frecuentemente involucrado en fracturas por insuficiencia pero no es el único. La primera descripción de fractura por insuficiencia sacra es atribuida a Lourie en $1982^{3,4}$

La población de mayor riesgo para fractura por insuficiencia pélvica (FIP) son mujeres postmenopáusicas que tienen osteoporosis y han recibido radioterapia. Otros factores predisponentes son el uso de corticoides, hormona tiroidea sustitutiva, enfermedades del tejido conectivo, diabetes mellitus, quistes de Tarlov, enfermedades metabólicas óseas, displasia fibrosa, obesidad, multiparidad, deformidades de la columna, excesiva anteversión pélvica e incluso embarazo ${ }^{5,6}$. Los últimos cinco factores, hacen referencia al exceso de peso que debe soportar una pelvis que ya está maltrecha. El resto de factores de riesgo hacen referencia a la propia calidad del hueso.

La incidencia de FIP está infraestimada y muchos casos quedan sin diagnóstico. Es muy probable que algunos sean asintomáticos o provoquen dolor leve que hace que el paciente no acuda a un servicio médico. Algo parecido a lo que ocurre con las fracturas vertebrales osteoporóticas que se considera se diagnostican $1 / 3$ del total.

Otra causa del infradiagnóstico es el hecho que en los estudios de TC y RM se busque enfermedad recurrente bien sea intestinal, genitourinaria o prostática, pasando desapercibida la afectación ósea no buscada.

El objetivo de nuestra presentación es recordar la existencia de esta enfermedad, más frecuente de lo que uno pudiera pensar, conocer los patrones clínicos y radiográficos, evitando confundir su diagnóstico y con ello realizar pruebas diagnósticas y tratamientos innecesarios.

\section{Caso Clínico}

Paciente de 73 años, que acudió a urgencias en Mayo de 2017 por dolor en región lumbar irradiado hasta pie derecho de cuatro días de evolución, sin traumatismo previo. Refería que el dolor le impedía caminar. La exploración neurológica era normal. No presentaba dolor a la palpaciónpercusión de apófisis espinosas lumbares ni sacro. La familia refería que el paciente sufría muchas caídas.
Antecedentes médicos: Cáncer de próstata diagnosticado y tratado en otro centro en 2013. La neoplasia de próstata se clasificó como Adenocarcinoma de alto riesgo Gleason 8 $(4+4)$ cT3b cN1 M0. Se perfiló un plan de tratamiento consistente en radioterapia y hormonoterapia. La hormonoterapia se inició en septiembre de 2013 y finalizó en Marzo de 2016 (30 meses, debido al alto riesgo). La radioterapia consistió en 36 sesiones, que se extendieron desde diciembre de 2013 hasta febrero de 2014, sobre cadena ganglionar ilíaca interna derecha, pelvis hasta L5$\mathrm{S} 1$, próstata y vesículas seminales. Además, colocación de 4 semillas de oro en glándula prostática vía transrectal ecodirigida.

Otros antecedentes incluían fractura de 1/3 proximal de húmero (tratado con osteosíntesis con clavo intramedular hacía 2 años), escoliosis lumbar degenerativa, estenosis de canal lumbar y espondiloartrosis.

En esta visita a urgencias la radiografía simple de pelvis fue normal (Fig. 1) y el paciente fue diagnosticado de lumbociatalgia aguda, y tratado con antiinflamatorios, analgésicos y relajantes musculares.

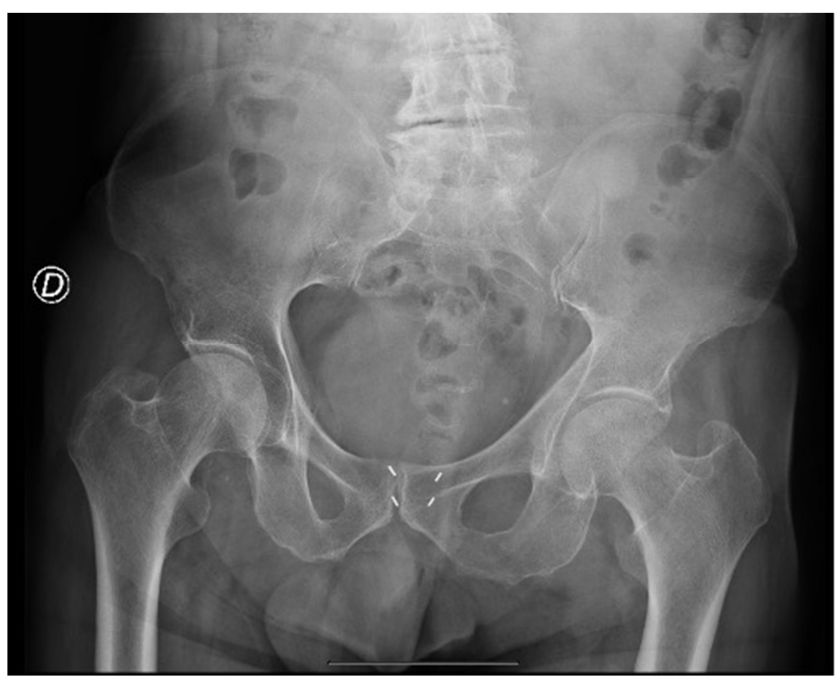

Figura 1: Radiografía anteroposterior de pelvis (Mayo 2017). Estudio radiológico dentro de la normalidad, sin alteraciones óseas relevantes.

Dos meses después acudió de nuevo a urgencias. Refería haber tropezado cinco días antes en la ducha sin llegar a caer al suelo. Presentaba dolor en nalga y región inguinal izquierda. Prácticamente no movía la pierna izquierda por dolor, pese a los analgésicos habituales pautados desde hacía tiempo. Presentaba edema llamativo en la extremidad inferior izquierda y pérdida de funcionalidad progresiva en las últimas semanas. No presentaba síndrome constitucional.

La radiografía simple de pelvis y caderas en ese momento demostraba desaparición de la epífisis proximal del fémur izquierdo como hallazgo más destacado (Fig. 2). Se observaba también una fractura de la sínfisis púbica. 
Además, el hueso ilíaco izquierdo era claramente patológico. La línea iliopectínea izquierda estaba interrumpida con uno o varios fragmentos óseos libres. Se apreciaba ensanchamiento del hueso ilíaco izquierdo con "fallas" osteolíticas (superpuestas con restos fecales colónicos). El área acetabular y supracetabular izquierda estaban desestructuradas.

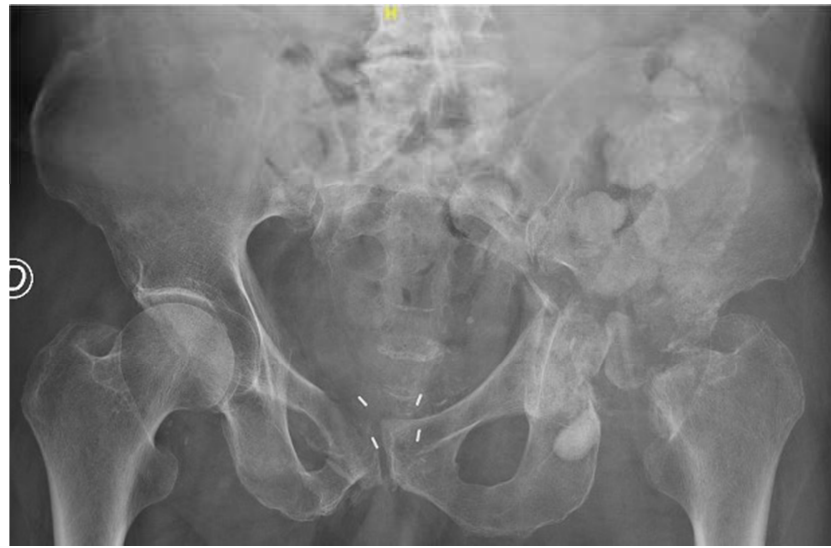

Figura 2: Radiografía anteroposterior de pelvis (Julio 2017). Desestructuración ósea del acetábulo izquierdo y extensa osteolisis de la epífisis proximal del fémur izquierdo. Hueso ilíaco de aspecto patológico, ensanchado y con presencia "fallas" osteolíticas. Fractura de la sínfisis púbica e interrupción de la línea iliopectínea izquierda con fragmentos óseos libres.

La enfermedad prostática en su última revisión, un mes antes de esta nueva visita a urgencias, se encontraba en estadio localizado, no existía evidencia de recaída, y el PSA era normal.

Dados los hallazgos en imagen el paciente ingresó en Medicina Interna-Oncología por sospecha de metástasis óseas múltiples.

La analítica en ese momento era: hemoglobina 10.50, hematocrito $32,30 \%$. Leucocitos 13,50 neutrófilos $85,20 \%$, urea y crea normales. Glucemia normal. VSG 31. Albumina 2.6. La proteína $C$ reactiva osciló durante su ingreso entre 47 y 168.

Se realizó estudio serológico para Coxiella burnetti, Brucela y Borrelia burgdorferi, Leishmania y tuberculosis, siendo negativo en todos los casos.

Durante su hospitalización se realizaron varias pruebas complementarias, entre ellas TC de pelvis y caderas. Las imágenes de TC mostraban marcada irregularidad cortical, amplias erosiones, osteolisis con severa destrucción ósea en acetábulo y hueso ilíaco izquierdo; osteolisis con fractura en hueso iliaco y pubis derechos, esclerosis difusa en el sacro y aumento de las partes blandas adyacentes a la cadera izquierda (Fig. 3,4 y 5).

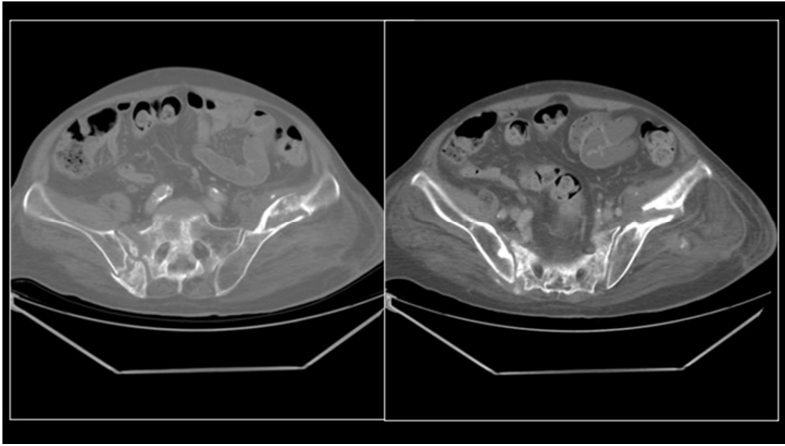

Figura 3: Tomografía computarizada (TC). Ventana ósea. Imágenes en plano axial. Se observa marcada irregularidad cortical, alteración difusa de la densidad ósea, en ambos iliacos y en sacro. Erosiones y osteolisis con fracturas de ambos huesos ilíacos.

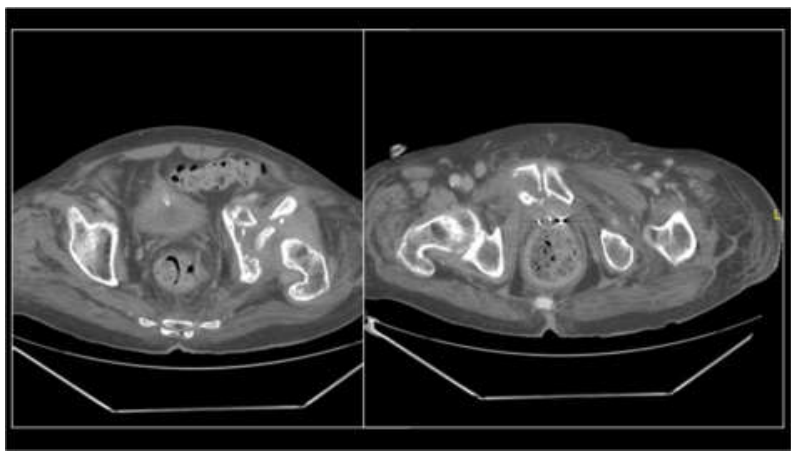

Figura 4: Tomografía computarizada (TC). Ventana ósea. Imágenes en plano axial. Destaca la extensa osteolisis con severa destrucción ósea en acetábulo y hueso ilíaco izquierdo. Fractura en hueso iliaco y pubis derechos. Importante destrucción articular de la cadera izquierda y aumento de las partes blandas adyacentes.

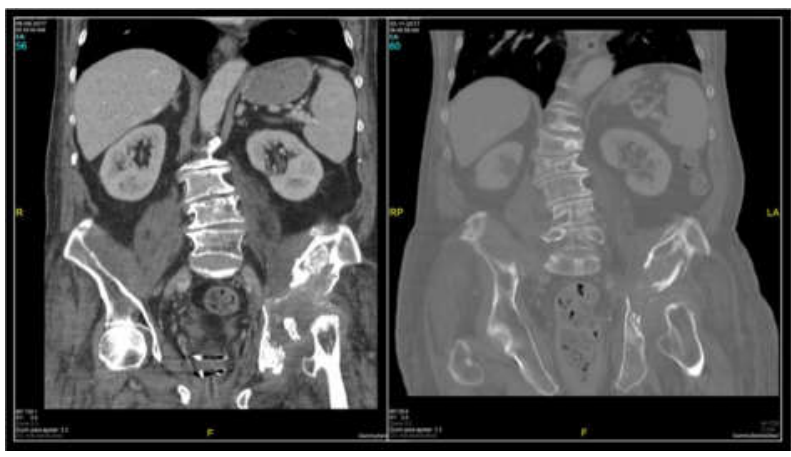

Figura 5: Tomografía computarizada (TC). Ventana ósea. Se observan los hallazgos referidos, en imágenes en plano coronal. 
En resumen, la sospecha radiológica era de metástasis óseas múltiples y fracturas patológicas a distintos niveles en la pelvis.

Se practicó estudio histológico mediante punción-biopsia hasta en dos ocasiones al no ser concluyente la primera vez. Según el informe de la segunda biopsia la extensión estaba constituida por celularidad inflamatoria dispersa, mayoritariamente linfocitaria con algunos macrófagos. No se observaron células epiteliales. Se programó una nueva biopsia, esta vez a cielo abierto cuyo resultado fueron signos de remodelación ósea (reabsorción y formación) asociadas a fibrosis sinovial. No se observó expresión nuclear de B-catenina en el tejido fibroso, infiltración tumoral ni granulomas. Su aspecto morfológico sugería la posibilidad de una fibromatosis (Fig. 6 y 7 ).

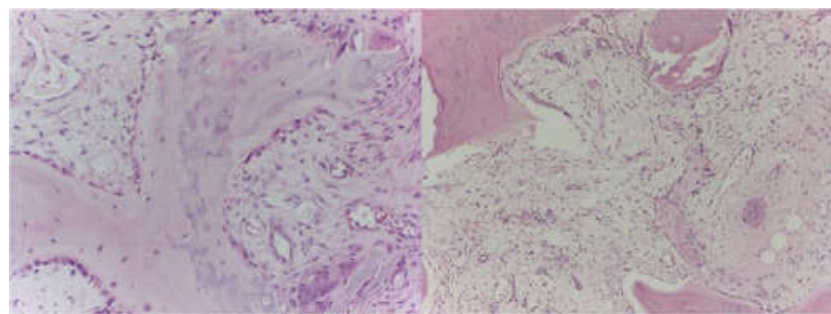

Figura 6: Imagen histológica. H.E. 10x. Celularidad inflamatoria dispersa, mayoritariamente linfocitaria con algunos macrófagos.

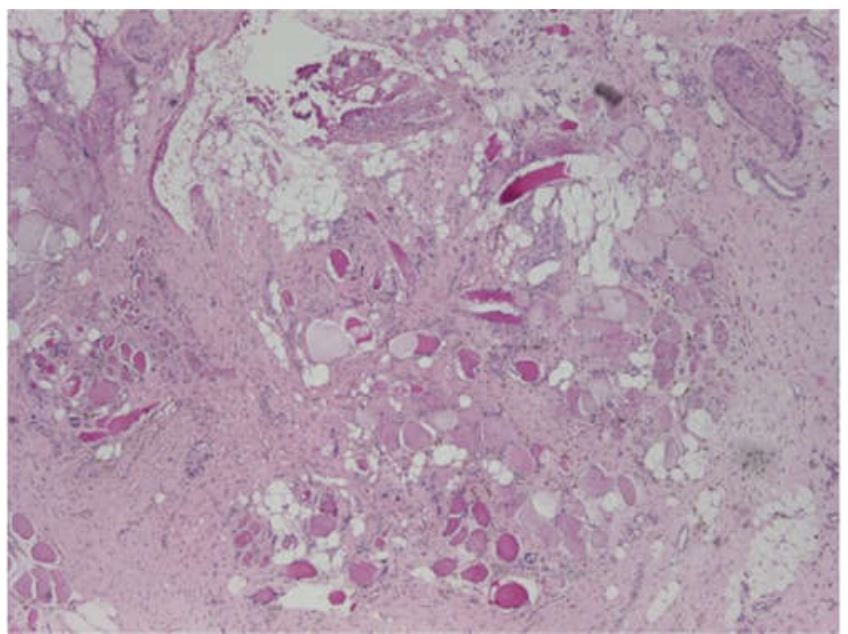

Figura 7: Imagen histológica. H.E. 10x. Signos de remodelación ósea (reabsorción y formación) asociadas a fibrosis sinovial.

El rastreo óseo mostraba una distribución heterogénea en hemipelvis y porción proximal de fémur izquierdo, con alteración de la morfología tanto del hueso ilíaco como de la articulación coxofemoral, todo ello en relación con lesiones líticas y fracturas. También se observó depósito del radiofármaco de morfología lineal en articulación sacroilíaca derecha y sacro, sugestivos de fracturas (Fig. 8).
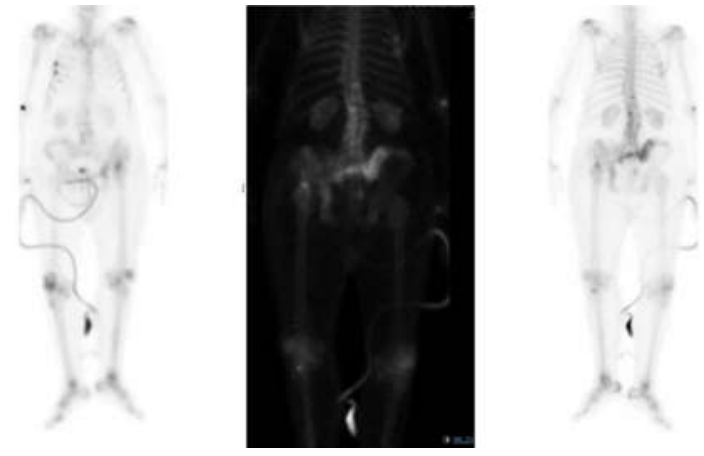

Figura 8: Gammagrafía con Tecnecio 99. Presencia del "signo Honda" incompleto a nivel de cuerpo y alas sacras.

El paciente permaneció en la unidad de medicina interna varias semanas. El dolor poco a poco fue atenuándose y mejorando el estado general. Finalmente se decidió derivar al paciente a un hospital de crónicos para continuar con la rehabilitación ya iniciada, y de allí a una residencia de ancianos, donde vive actualmente.

Posteriormente a su alta hospitalaria el paciente fue estudiado en consultas externas de traumatología. El dolor había disminuido bastante. El paciente podía incluso ponerse de pie con la ayuda de un andador.

Se realizó radiografía de pelvis y caderas, analítica ordinaria y densitometría.

La densitometría ósea revelaba estas cifras: Cadera, Tscore: $-2,9$ Columna, T-score: $-1,5$

Analítica: vitamina D 13,69 PTH: 47,8 Calcio corregido por albúmina: 9 , Calciuria normal.

Se analizaron de nuevo minuciosamente las imágenes previas de radiografía simple, TC, PET-TC y Gammagrafía, así como la analítica y densitometría ósea.

Con todo ello, y una vez descartado con el estudio histológico la presencia de enfermedad maligna primaria o secundaria, se diagnosticó por exclusión, de osteítis por radiación y fracturas múltiples por insuficiencia pélvica.

\section{Discusión}

La fractura por insuficiencia pélvica secundaria a radioterapia es una complicación raramente vista, sobre todo desde el uso de los equipos de megavoltaje, pero desafortunadamente no ha desaparecido ${ }^{7}$. La incidencia de fracturas pélvicas sintomáticas en pacientes irradiados, oscila entre $3-5 \%$. Sin embargo, cuando se estudian activamente a los pacientes asintomáticos la incidencia aumenta hasta $34 \%^{8}$. Se ha encontrado una incidencia para el carcinoma anal del $14 \%$, para el rectal de $8,7 \%$ para el de cérvix $8,2 \%^{9}$, y $6,8 \%$ en cáncer de próstata ${ }^{10}$. Otro estudio detectó $89 \%$ de pacientes, usando $\mathrm{RM}^{11}$, y otro publicó $34 \%$ empleando gammagrafía ${ }^{8}$.

La atrofia ósea postradiación es dosis dependiente y sucede cuando las dosis exceden de $40 \mathrm{~Gy}^{12,13}$. Aspectos técnicos que incrementan el riesgo de fractura por insuficiencia incluyen el número de campos tratados por día, el uso de terapia de ortovoltaje y la alta dosis diaria por 
fracción ${ }^{13}$. El lapso de tiempo entre radioterapia y aparición de las fracturas varía entre 2 semanas a 8 años y es impredecible, aunque la mayoría de los casos ocurren dentro de los tres primeros años ${ }^{12}$.

Hay que diferenciar lo que son cambios histológicos provocados en el hueso por la radioterapia y que se conocen como osteítis por radiación, de la fractura por insuficiencia como resultado final de todo el proceso. El término osteítis por radiación se refiere a una respuesta inflamatoria en los vasos sanguíneos, tejido nervioso y células óseas que pueden conducir a una fractura ósea. La histología de las lesiones se caracteriza por hemorragia, fibrosis, fragmentos óseos necróticos y crecimiento de hueso trabecular y cartílago. La necrosis avascular puede afectar a cualquier hueso irradiado, probablemente debido a lesiones en la microvascularización del hueso maduro ${ }^{13}$.

La forma de presentación clínica más frecuente es dolor de espalda o nalga, en el $64 \%$ y $43 \%$ respectivamente, aunque hasta un $22 \%$ de los casos es asintomático en el momento del diagnóstico8. Aproximadamente el $80 \%$ de pacientes se quejan de sensibilidad sacra y el $14 \%$ tienen síntomas neurológicos6. Además estos pacientes pueden tener hematuria, sangrado rectal u obstrucción intestinal ${ }^{14}$. Sin embargo la presencia de dolor lumbar bajo, púbico o de ingle, o dolor radicular en la extremidad inferior puede conducir al erróneo diagnóstico pero mucho más habitual de enfermedad degenerativa discal, estenosis espinal, artrosis de cadera o fractura por compresión vertebral6, diagnósticos que pueden superponerse y contribuir a la confusión. Algunos pueden llegar a tener dolor severo y en ellos suele coincidir la presencia de múltiples sitios de fractura15, como es el caso de nuestro paciente. Sólo $50 \%$ de pacientes cuyas fracturas por insuficiencia fueron detectadas por técnicas de imagen, eran sintomáticos ${ }^{15}$.

La apariencia radiográfica de osteítis por radiación varia con los sitios anatómicos afectados. Así, en el cráneo, mandíbula o falanges se aprecia osteopenia como única anormalidad $^{16}$. El hueso irradiado puede observarse osteopénico aproximadamente un año tras la radioterapia y suelen apreciarse intentos de reparación ósea con áreas mixtas de esclerosis y osteopenia a los 2-3 años ${ }^{11}$. Pueden detectarse cambios esqueléticos en sacro, cadera, ramas púbicas, sínfisis púbica, ilion y columna lumbar baja ${ }^{13,14}$.

Es habitual que la radiografía simple de pelvis sea normal en el momento de la primera consulta. Es más probable diagnosticarla mediante TC o gammagrafía, en esos momentos iniciales. En otros casos más evolucionados, las radiografías de pelvis, sacro y columna lumbar muestran bandas escleróticas, interrupción de la cortical y líneas de fractura, sin embargo los cambios sutiles no son vistos normalmente ${ }^{17}$. Algunas veces como en nuestro caso clínico, los fenómenos de reparación ósea pueden demostrar una imagen agresiva que simula enfermedad maligna $^{18}$.

La fractura de sacro es la más frecuentemente publicada ${ }^{2}$, porque el desarrollo de la fractura tiene relación con la carga de peso. El sacro, sacroilíacas y áreas mediales de los huesos ilíacos son las estructuras del cuerpo que soportan mayor carga de peso. Puede asociarse fractura de huesos púbicos o acetábulo a la fractura sacra, pero raramente se presentan éstas últimas como una fractura aislada, de forma que el fracaso mecánico del sacro es el causante de otras subsecuentes fracturas ${ }^{11}$. También se han publicado fracturas del cuello de fémur y de la región subtrocantérea $^{19,20}$.

El diagnóstico de fractura por insuficiencia no es fácil habitualmente $y$ lo es menos en un esqueleto osteopénico $^{21}$. Lo habitual no es encontrarse como en este caso, fracturas múltiples en distintos momentos evolutivos. Es más frecuente la fractura vertical en el ala sacra y orientadas paralelamente a la articulación sacroilíaca. Posteriormente pueden aparecer trazos de fracturas secundarios horizontales. La presencia de fracturas asociadas en columna o pelvis, ayudan al diagnóstico de la fractura por insuficiencia de sacro ${ }^{22,23,24}$. Habitualmente el TC confirma la ó las fracturas y elimina la posibilidad de un proceso destructivo.

La gammagrafía ósea sigue siendo uno de los exámenes más sensibles en la detección de fracturas por insuficiencia sacra (FIS). El típico signo llamado "Honda" o patrón $\mathrm{H}$ se considera diagnóstico de FIS, sin embargo es visible de forma completa sólo en el $40 \%$ de los casos $^{25}$. La presencia del signo "H" o una variante, con evidencia de otras fracturas regionales o con ausencia de enfermedad metastásica debería conceder una fuerte evidencia de fractura por insuficiencia sacra. La gammagrafía con tecnecio 99 tiene una sensibilidad de $96 \%$ para la detección de FIS $^{26}$

La RM es una técnica de imagen muy sensible para detectar las alteraciones precoces de la médula ósea. Los patrones típicos de osteítis postradiación en pelvis se describen como disminución difusa de la intensidad de señal en el hueso en las secuencias potenciadas en T1 y una intensidad de señal intermedia o alta en secuencias potenciadas en T2 y STIR ${ }^{11}$. La RM con frecuencia muestra una línea de fractura en una localización típica de las fracturas por insuficiencia ${ }^{11,19,22}$. En estos casos identificaremos la presencia de imágenes lineales hipointensas en secuencias potenciadas en T1, T2 y STIR ${ }^{27}$. La secuencia STIR es la ideal cuando se asocian osteítis por radiación con fractura por insuficiencia ${ }^{27}$. Estas anomalías también son típicas de la enfermedad metastásica, pero la presencia de líneas de fractura y sin presencia de masa focal, ayuda a establecer el diagnóstico. Las fracturas por insuficiencia no son asociadas con lesiones osteolíticas ni masa de partes blandas, las cuales si son comunes en enfermedad metastásica ${ }^{23}$.

Los hallazgos de osteolisis son poco frecuentes y se deben a una fractura por insuficiencia paraarticular asociada con osteopenia y radioterapia. La osteólisis se caracteriza en radiografía por cambios destructivos que progresan rápidamente en la sínfisis del pubis o la articulación sacroilíaca. La presencia de un callo óseo excesivo e ineficaz sugiere un intento de curación de la fractura con respuesta osteoblástica apropiada pero con una respuesta osteoclástica desproporcionada ${ }^{19}$. Los hallazgos de la osteolisis en la RM son como una hendidura, con un área 
de señal brillante alargada que sugiere una "acumulación de líquido" dentro de la brecha de fractura y la ausencia de realce de contraste ${ }^{28}$.

El diagnóstico diferencial debe incluir: enfermedad metastásica, segunda neoplasia primaria, sarcoma postradiación, hiperparatiroidismo, infecciones como tuberculosis y fibromatosis abdominal.

La enfermedad metastásica es excepcional en un terreno irradiado11 y tiende a presentar un patrón aleatorio en la gammagrafía en todo el esqueleto ${ }^{29}$.

El diagnóstico de segunda neoplasia primaria, así como el sarcoma postradiación queda eliminada por la ausencia de tumor en las biopsias realizadas. Además, los sarcomas postradiación se ven en menos de $0.1 \%$ de los pacientes que han recibido radioterapia y sobreviven 5 años ${ }^{30}$. Para que un tumor sea clasificado como inducido por radiación, la lesión debe originarse en el campo irradiado, los hallazgos histológicos deben de ser diferentes del tumor primitivo y el tiempo entre la radioterapia y la aparición del tumor debe ser suficientemente largo, normalmente más de 10 años $^{29}$.

Siempre que tengamos osteopenia-osteoporosis y fracturas patológicas, habrá que pensar en la posibilidad de estar ante un hiperparatiroismo. Para ello la hormona PTH debería estar elevada (tanto en formas primarias como secundarias) y en nuestro paciente era rigurosamente normal, así como la calcemia y la calciuria. Tampoco tenía otros hallazgos radiográficos ni clínicos característicos de tal enfermedad.

El diagnóstico definitivo de tuberculosis osteoarticular es anatomopatológico y microbiológico y fue descartado en este paciente.

La fibromatosis abdominal se suele manifestar como una masa cuya sintomatología dependerá de la localización; lo habitual es que ocasione obstrucción intestinal e hidronefrosis. Es más frecuente en mujeres de tercera o cuarta década, y no provoca fracturas múltiples. Aun así, los anatomopatólogos atendiendo exclusivamente a criterios histológicos pensaron en ésta posibilidad.

En este paciente las imágenes radiográficas iniciales fueron preocupantes al tener en cuenta sus antecedentes médicos. Precisamente el diagnóstico de presunción fue el de metástasis de adenocarcinoma de próstata. Posteriormente la descripción de las imágenes de TC, aumentaba la sospecha de tales metástasis múltiples diseminadas por la pelvis, asociada a diversas fracturas patológicas. Sin embargo, ésta sospecha no fue refrendada por la anatomía patológica. Después de varias semanas de ingreso hospitalario y sucesivas TC de control de las lesiones, sin grandes cambios, el paciente fue dado de alta sin diagnóstico, en cuanto mejoró sustantivamente del dolor.
Las fracturas por insuficiencia tras radiación son más difíciles de tratar; el reemplazo protésico de cadera se ha asociado con alto riesgo de complicaciones que incluyen infección e insuficiencia mecánica ${ }^{31}$.

\section{Conclusión}

Muchos de los tumores intrapélvicos malignos se ven en mujeres postmenopáusicas en las cuales ya existe una osteopenia u osteoporosis previa a la radioterapia. Es muy importante conocer los factores de riesgo de fragilidad ósea así como de causas secundarias de osteoporosis y de iatrogenia farmacológica. Así por ejemplo nuestro paciente fue tratado con inhibidores de la gonadotrofina para la neoplasia de próstata y se sabe que tal tratamiento produce una caída de la masa ósea durante el primer año en torno a un $2,5-5 \%$ y aumenta el riesgo de fractura vertebral y de cadera a los 5 años en torno a $20-50 \%{ }^{32}$. Además el paciente había sufrido previamente una fractura de tercio proximal de húmero por caída de propia altura, la cual debió ya ser identificada y evaluada como una fractura por fragilidad y probablemente tratada con fármacos antirresortivos y suplementos de calcio y vitamina $\mathrm{D}$.

En los pacientes que sufren una neoplasia urogenital, de colon o de próstata y van a recibir radioterapia como parte del tratamiento, es recomendable hacer un seguimiento mediante densitometría y estudio analítico del metabolismo óseo, incluso previo a cualquier fractura por fragilidad. A nuestro paciente, con una buena función renal, se le pautó alendronato semanal vía oral, además de suplementos de calcio y de vitamina $\mathrm{D}$ a dosis de choque iniciales.

Probablemente el hecho de confiar excesivamente en las descripciones radiográficas y de TC, hizo disminuir la posibilidad de buscar otros diagnósticos y contribuyó definitivamente a la demora en el diagnóstico verdadero. Es importante conocer la existencia y la incidencia de FIP tras radioterapia con el fin de evitar el erróneo diagnóstico de metástasis óseas, infecciones o tumores primarios diferentes al primitivo. Es asimismo importante conocer los patrones de imagen de la osteítis por radiación en radiografía, gammagrafía, TC y $\mathrm{RM}$, que será la causante de las fracturas posteriores, para evitar al paciente demoras en el diagnóstico, así como biopsias e incluso tratamientos equivocados. 
FERNANDEZ GABARDA R y COLS. Osteítis por radiación y fracturas por insuficiencia pélvica en paciente con adenocarcinoma de próstata tratado con radioterapia. Aspectos clínicos y radiográficos de la enfermedad.

\section{Bibliografía}

1. Ewing, J. Radiation osteítis. Incidence and possible aetiological factors in the development of pelvic insufficiency fractures following radical radiotherapy. Acta Radiol 1926; 6:399-412.

2. Dongryul Oh, Seung Jae Huh. Insufficiency fracture after radiation therapy. Radiat Oncol J. 2014 Dec; 32(4):213-20.

3. Lourie H. Spontaneous osteoporotic fracture of the sacrum: an unrecognized syndrome of the elderly. JAMA 1982; 248:715-7.

4. Aditya V. Maheshwari MD, Jeremy S. Frank MD, J. David Pitcher Jr. MD, H. Thomas Temple MD . Low Backache in a 70-year-old Woman. Clin Orthop Relat Res 2009; 467:596-602

5. Bliss P, Parsons CA, Blake PR. Incidence and possible aetiological factors in the development of pelvic insufficiency fractures following radical radiotherapy. Br J Radiol 1996; 69:548-554.

6. Grasland A, Pouchot J, Mathieu A, Paycha F, Vinceneux P. Sacral insufficiency fractures: an easily overlooked cause of back pain in elderly women. Arch Intern Med 1996; 156:668-74.

7. Howland, W J, Loeffler, K, Starchman, D E and Johnson, R G. Postirradiation atrophic changes of bone and related complications, Radiology 1975;117: 677-85.

8. Abe H, Nakamura M, Takahashi S, Maruoka S, Ogawa Y, Sakamoto K. Radiation-induced insufficiency fractures of the pelvis: evaluation with 99mTc-methylene diphosphonate scintigraphy. Am J Roentgenol 1992; 158:599- 602.

9. Baxter NN, Habermann EB, Tepper JE, Durham SB, Virnig BA. Risk of pelvic fractures in older women following pelvic irradiation. JAMA2005; 294:2587-93.

10. Igdem S, Alco G , Ercan T, Barlan M, Ganiyusufoğlu K, Unalan B, et al. Insufficiency fractures after pelvic radiotherapy in patients with prostate cáncer. Int J Radiation Oncology Biol Phys 2010;77(3):818-23.

11. Blomlie V, Rofstad EK, Talle K, Sundfor K, Winderen M, Lien HH. Incidence of radiation-induced insufficiency fractures of the female pelvis: evaluation with MR imaging. AJR Am J Roentgenol 1996;167:1205-10.

12. Fu AL, Greven KM, Maruyama Y. Radiation osteitis and insufficiency fractures after pelvic irradiation for gynecologic malignancies. Am $J$ Clin Oncol 1994;17:248-54

13. Mumber MP, Greven KM, Haygood TM. Pelvic insufficiency fractures associated with radiation atrophy: clinical recognition and diagnostic evaluation. Skeletal Radiol 1997; 26:94-9.

14. Peh, W C G, Khong, P L Sham, J S T, Ho WY,Yeung HWD. Sacral and pubic insufficiency fractures after irradiation of gynaecological malignancies. Clin Oncol 1995; 7:117-22.

15. Oh D, Huh SJ, Nam H, Park W, Han Y, Lim DH, et al. Pelvic insufficiency fracture after pelvic radiotherapy for cervical cancer: analysis of risk factors. Int J Radiat Oncol Biol Phys 2008; 70:1183-8.

16. Bragg, D G, Shidnia, H, Chu, F C H and Higinbotham, N L. The clinical and radiological aspects of radiation osteitis. Radiology 1970; 97: 103-11.

17. Newhouse KE, el-Khoury GY, Buckwalter JA. Occult sacral fractures in osteopenic patients. J Bone Joint Surg Am 1992; 74:1472-7.

18. Peh WC, Khong PL, Yin Y, Ho WY, Evans NS, Gilula LA, et al. Imaging of pelvic insufficiency fractures. Radiographics 1996; 16:33548.

19. Kwon JW, Huh SJ, Yoon YC, Choi SH, Jung JY, Oh D, et al. Pelvic bone complications after radiation therapy of uterine cervical cancer: evaluation with MRI. AJR Am J Roentgenol 2008; 191:987-94.

20. Epps HR, Brinker MR, O'Connor DP. Bilateral femoral neck fractures after pelvic irradiation. Am J Orthop (Belle Mead NJ) 2004; $33: 457-60$

21. Leroux JL, Denat B, Thomas E, Blotman F, Bonnel F. Sacral insufficiency fractures presenting as acute low-back pain: biomechanical aspects. Spine 1993; 18:2502-6.

22. Blomlie V, Lien HH, Iversen T, Winderen M, Tvera K. Radiation-induced insufficiency fractures of the sacrum: evaluation with MR imaging. Radiology 1993; 188:241-4. 
FERNANDEZ GABARDA R y COLS. Osteítis por radiación y fracturas por insuficiencia pélvica en paciente con adenocarcinoma de próstata tratado con radioterapia. Aspectos clínicos y radiográficos de la enfermedad.

23. Cooper KL, Beabout JW, Swee RG. Insufficiency fractures of the sacrum. Radiology 1985; 156:15-20.

24. Schneider R, Yacovone J, Ghelman B. Unsuspected sacral fractures: detection by radionuclide bone scanning. AJR Am J Roentgenol 1985;144:337-41.

25. Blake SP, Connors AM. Sacral insufficiency fracture. Br J Radiol 2004; 77:891-6.

26. Fujii M, Abe K, Hayashi K, Kosuda S, Yano F, Watanabe S, et al. Honda sign and variants in patients suspected of having a sacral insufficiency fracture. Clin Nucl Med 2005; 30:165-9.

27. Yoshioka H, Nakano T, Kandatsu S, Koga M, Itai Y, Tsujii H. MR imaging of radiation osteitis in the sacroiliac joints. Magn Reson Imaging 2000; 18:125-8.

28. Seo GS, Aoki J, Karakida O, Sone S, Ishii K. Ischiopubic insufficiency fractures: MRI appearances. Skeletal Radiol 1997; 26:705-710.

29. Kim HJ, Boland PJ, Meredith DS, Lis E, Zhang Z, Shi W, et al. Fractures of the sacrum afterchemoradiation for rectal carcinoma: Incidence, risk factors, and radiographic evaluation. Int J Radiation Oncol Biol Phys 2012; 84(3):694-9.

30. Tountas AA, Fornasier VL, Harwood AR, Leung PM. Postirradiation sarcoma of bone: a perspective. Cancer 1979; 43:182-7.

31. Massin P, Duparc J. Total hip replacement in irradiated hips: a retrospective study of 71 cases. J Bone Joint Surg Br 1995; 77:847-52.

32. Bienz M, Saad F. Androgen-deprivation therapy and bone loss in prostate cancer patients: a clinical review. Bonekey Rep 2015; 4:716. 